\title{
Xenobiotics-Induced Liver Damage Is Biochemically Abrogated by Treatment with Lipophilic Extracts of Moringa oleifera in Vivo
}

\author{
Maxwell Omabe ${ }^{1,2^{*}}$, Kenneth Nwobini Omabe³, David Igwe4, Ogbonnaya Chukwu John², \\ Samuel Kalu Uchenna ${ }^{2}$, Sunday Elom ${ }^{5}$
}

${ }^{1}$ Department of Oncology, Cancer Research Division, Immunology Group, University of Saskatchewan,

Saskatoon, Canada

${ }^{2}$ Diagnostic Molecular Biology Research Group, Molecular Pathology and Immunology Division, Chemical Pathology \& Histopathology Unit, Department of Medical Laboratory Sciences, School of Biomedical Science, Faculty of Health Science, Ebonyi State University, Abakaliki, Nigeria

${ }^{3}$ Department of Molecular Biology, Faculty of Natural Sciences, Federal University, Abakaliki, Nigeria

${ }^{4}$ Department of Trauma and Orthopaedic Rotherham NHS Trust, Rotherham, UK

${ }^{5}$ Department of Medical Biochemistry, Faculty of Basic Medical Sciences, Federal University, Abakaliki, Nigeria

Email: *Maswello2002@yahoo.com

How to cite this paper: Omabe, M., Omabe, K.N., Igwe, D., John, O.C., Uchenna, S.K. and Elom, S. (2018) Xenobiotics-Induced Liver Damage Is Biochemically Abrogated by Treatment with Lipophilic Extracts of Moringa oleifera in Vivo. Health, 10, 313-325. https://doi.org/10.4236/health.2018.103025

Received: March 8, 2018

Accepted: March 19, 2018

Published: March 22, 2018

Copyright ( 92018 by authors and Scientific Research Publishing Inc. This work is licensed under the Creative Commons Attribution International License (CC BY 4.0).

http://creativecommons.org/licenses/by/4.0/ (c) (i) Open Access

\begin{abstract}
Context Drug-induced hepatotoxicity represents a significant proportion of liver disease cases. Currently, there is no effective treatment. To date efforts to identify treatment regimen that can reverse progressive damage have not been successful. We have previously shown that extract from Moringa ( $M$ ) oleifera possesses clinically relevant antidiabetic and electrolyte modulators. Objective The aim of the current studies is to create experimental model of xenobiotic induced liver damage and investigate if treatment with lipophilic extract of $M$. oleifera could biochemically reverse progressive liver damage. Materials and Method For two groups of healthy rats, 7 in each group received $200 \mathrm{mg}$ of extract or vehicle twice daily for 14 days. Acute toxicity, hepatotoxicity and hematologic/endothelial toxicity were monitored. Then 30 rats weighing 130 $200 \mathrm{~g}$ received repeated dose of acetaminophen (xenobiotics) $(640 \mathrm{mg} / \mathrm{kg}$ ) for 5 days. Hepatotoxicity was confirmed biochemically by an established protocol. Treatment with $M$. oleifera extract resulted in mean weight of $132.2 \pm 5.05$ compared to the control with $134.1 \pm 5.08(\mathrm{P}>0.8115)$ among the healthy rats. Their LDH levels were $170.7 \pm 13.02$ and $133.8 \pm 7.17$ ( $\mathrm{P}>0.0698)$ for controls group, while the mean serum (ALT) level was $12.4 \pm 1.2$ or $25.6 \pm 5.644$ $(\mathrm{P}<0.01)$ for controls group. However, treatment of rats with hepatitis using
\end{abstract}


lipophilic extract of $M$. oleifera resulted in 100\% biochemical recovery from hepatitis compared to the control group $(\mathrm{P}<0.0006)$. Conclusion This study strongly indicates that treatment with lipophilic extract of $M$. oleifera could effectively and biochemically abrogate xenobiotics induced liver damage in animal model.

\section{Keywords}

Phytopharmaceuticals, Phytomedicine, Drug Discovery, Inflammation, Alanine-Aminotransferase, Haematological Toxicity, Lactate Dehydrogenase, Hepatitis

\section{Introduction}

Liver is an organ of paramount importance responsible for metabolic functions including detoxification of exogenous and endogenous challenges such as xenobiotics [1] [2]. In keeping with this function, some xenobiotics including therapeutic drugs like analgesics cause drug induced liver injury (DILI), toxicity, and damages; this is often characterised with hepatocellular swelling, inflammation and increased in biochemical marker of hepatocellular necrosis [3], and represents a major source of morbidity and mortality [4]. DILI due to intrinsic hepatotoxicity following acetaminophen overdose accounts for the majority of cases of drug-induced acute liver failure [5]. Currently, there is no available cure for severe progressive liver damage. Common treatment for total liver failure is liver transplantation, but patients who are unable to afford this treatment would definitely die of the disease. In fact, current evidence shows that death due to liver disease is on the rise across the world with some data suggesting that more than 130 million people are currently living with liver disease. In next few years, this figure is likely to quadruple.

Medical intervention for most liver diseases is generally based on symptomatic management of some underlying pathologies and complications, and life style modification. Some new forms of treatment have been reported with most of them being experimental.

As cause of death due to liver disease continues to rise worldwide, available treatment options are limited and some time very costly and unaffordable by majority of patients especially in developing countries. However, some of treatment options often fail to help about $50 \%$ of the patients whose hepatotoxicity and damage becomes worse, and many of them cannot get a transplant. They are often facing death due to treatment failure or inability to afford the cost. Abundant published evidence indicates that natural products or phytocompounds might provide a potential alternative therapeutics for liver disease. To investigate this, we created an animal model of both liver and metabolic syndrome; and previously showed that extract from $M$. oleifera had clinically relevant antidiabetic and electrolyte modulators [6] [7]. Here we propose that lipophilic extract from Moringa leaves may contain some bioactive compounds that may 
control drug induced hepatotoxicity and promote recovery of the liver after hepatic damage. First, we evaluated some associated biochemical or hematologic/endothelial marker of tissue toxicity and hepatotoxicity in healthy adult and in experimentally induced live disease in rats. We report that treatment with lipophilic extract of $M$. oleifera halted and reversed biochemical progression of acetaminophen-induced hepatotoxicity in animal model, and demonstrate that the crude extract promoted and enhanced biochemical recovery from drug-induced hepatotoxicity in murine model.

\section{Materials and Method}

\subsection{Plant Material}

Fresh mature leaves of $M$. oleifera were identified and confirmed by Senior Botanist in the Department of Biological Sciences, Edo University, and were subsequently supplied to our Group. The leaves were kept cold and protected from light during transportation and extraction processes. The leaves were dried under shade and then blended manually into smaller sizes. M. oleifera leaves (5 $\mathrm{kg}$ ) were extracted according to protocol described by Ghasi et al. [8]. The leaves were processed through cold solvent extraction by homogenizing with $25 \mathrm{~L}$ of acidified aqueous-ethanol solution containing $1 \%$ acetic acid and $50 \%$ ethanol. The extract was then filtered to remove the residue and concentrated by evaporating at $40^{\circ} \mathrm{C}$. The $\mathrm{pH}$ was then adjusted to 3.5 . Extract were subsequently air-dried by evaporation at ambient temperature. Fully dried extract were then weighed and stored in airtight containers at $-20^{\circ} \mathrm{C}$ till the time for further studies.

\subsection{Experimental Animal}

Among limitations for studying liver disease in human includes ethical issues and cost. For these reasons, we choose a translational approach and created a potent disease model. Animal models are commonly created to recapitulate certain aspects of corresponding human diseases and serve as important tool in studying disease mechanisms and for testing therapeutic strategies. In view of their experimental validity as previously reported [9]; Albino rat models have been predictive of clinical outcome in studying xenobiotics induced liver disease [9]. For this reason, we created drug induced liver disease in albino rat and undertook the following procedures. A written approved by the University ethical review board for use of animal in experiment was obtained before the start of the experiments. All the experiments were conducted in accordance with the Animal (Scientific Procedures) Act 1986 which are in strict compliance with the UKCCCR guidelines (2010) for the welfare of animals in experimental neoplasia [10]. In brief, adult's albino rats of both sexes and the same age group (8 - 12 weeks) weighing 130 - $200 \mathrm{~g}$ were obtained from the Pharmacological Laboratory of the University of Nigeria, Nsukka. The animals were housed at the animal house facility in the biochemistry department of Edo University. The rats 
were acclimatized for 2 weeks and feed with water and commercial live stock feed (grower). The regulatory standard for ethnical use of animals in scientific research in Nigeria was strictly observed.

\subsection{Therapy with M. oleifera Extract in Healthy Rat}

Healthy adult rats weighing between ( 8 - 12 weeks) weighing $130-200 \mathrm{~g}$ were used in this study, the animal were divided into 2 groups. The first group, 7 in number received repeated dose of $200 \mathrm{mg}$ of M. oleifera extract orally, according to the methods of Ghasi et al. [8] while the second group, also 7, received vehicle only for 14 days.

\subsection{Drug Induced Hepatitis in Rat}

Rats weighing between 130 and $200 \mathrm{~g}$ were used in this study, the animals received a repeated dose of normal saline and acetaminophen $(640 \mathrm{mg} / \mathrm{kg}$ suspended in $1 \%$ methylcellulose) otherwise referred to in this study as xenobiotics, administered orally in according to the method of Ghasi et al. [8], and hepatitis was confirmed by sustained high value of ALT.

\subsection{Treatment of Drug Induced Hepatitis Rats with Lipophilic Extract of M. oleifera}

Rats with confirmed hepatitis were divided into two groups, the first group received vehicle - normal saline $(10 \mathrm{ml} / \mathrm{kg})$ and the vehicle ( $1 \%$ methylcellulose, 13 $\mathrm{ml} / \mathrm{kg}$ ) orally and the second group was treated with M. oleifera extract (200 $\mathrm{mg} / \mathrm{kg}$, dissolved in $5 \mathrm{ml}$ saline with ( $1 \%$ methylcellulose, $13 \mathrm{ml} / \mathrm{kg}$ ) which was administered oral for 10 days.

\subsection{Collection of Blood Sample from the Rats}

Blood sample collections throughout the period of experiment were in accordance with the method of Baravalia and Chanda [11]. The collected blood samples were transferred into plain tubes or lithium heparin or potassium fluoride container which were appropriately labeled according to the identification label and were centrifuged at $3000 \mathrm{~g}$ for $15 \mathrm{~min}$ to separate plasma or serum from the whole blood. The separated plasma or serum was stored in refrigerator until when required for experiments or was analyzed immediately.

\subsection{Acute Toxicity Test}

The acute toxicity of the $M$. oleifera was evaluated in rats using the up and down procedure. Total of 6 rats (weight: 130 to $200 \mathrm{~g}$ ) received M. oleifera starting at 0.1 to $1 \mathrm{~mL} /$ rat orally by gavage. The animals were observed for toxic symptoms continuously for first $4 \mathrm{~h}$ after dosing. Finally, the number of survivors was noted after $24 \mathrm{~h}$. The animals were then maintained and observed for further 5 days. 


\subsection{Determination of Alanine Transaminase Level}

Aminotransferase levels are sensitive indicators of liver-cell injury and are helpful in recognizing hepatocellular diseases such as hepatitis. Aspartate aminotransferase is found, in decreasing order of concentration, in the liver, cardiac muscle, skeletal muscle, kidneys, brain, pancreas, lungs, leukocytes, and erythrocytes [12]. The highest level of alanine aminotransferase is in the liver, and levels of this enzyme are accordingly more specific indicators of liver injury. In health, aminotransferases are normally present in serum at low levels. The kinetic method was used for ALT determination. Alanine amino transferase catalyzes the transfer of amino acid from alanine to $\alpha$-ketoglutarate to form pyruvate and glutamate. The pyruvate formed is reduced to lactate in the presence of lactate dehydrogenase and NADH. This is then measured at $340 \mathrm{~nm}$.

$$
\begin{gathered}
\text { Alanine }+ \text { 2-oxoglutarate } \rightarrow \text { pyruvate }+ \text { L-Glutamate } \\
\text { Pyruvate }+\mathrm{NADH}+\mathrm{H}^{+} \mathrm{P}^{\mathrm{H}} 7.4 \rightarrow \text { Lactate }+\mathrm{NAD}+\mathrm{H}_{2} \mathrm{O}
\end{gathered}
$$

The spectrophotometer was initially blanked using distilled water, then 1000 $\mu \mathrm{l}$ of the commercially prepared reagent was added into a test tube using automatic pipette, after which $25 \mu \mathrm{l}$ of the sample was added with a gentle vertex. The optical density was determined according to the manufacturer's instruction and was measured at $340 \mathrm{~nm}$ wavelength.

\subsection{Determination of Lactate Dehydrogenase Level (LDH)}

LDH catalyzes the oxidation of lactate to pyruvate in the presence of NAD, which is subsequently reduced to NADH. The rate of NADH formation measured at $340 \mathrm{~nm}$ is directly proportional to serum LDH activity. A commercially prepared kit from Sigma UK was used following the manufacturers instruction. This involved addition of $1000 \mu \mathrm{l}$ of the reagent into a test tube, then $100 \mu \mathrm{l}$ of the sample into the same tube using automatic pipette and mixed gently.

The optical density was determined at $340 \mathrm{~nm}$ wavelength.

\subsection{Statistical Analysis}

The results are expressed as mean \pm SEM, $(\mathrm{N}=7)$. Statistical significance was determined by one-way analysis of variance with $\mathrm{P}<0.05$ considered significant. The analysis was performed by Prism software. Statistical analysis of the result obtained was done using mean \pm standard error of mean (S.E.M.).

\section{Result}

\subsection{M. oleifera Extract Did Not Cause Weight Changes; i.e. Weight Loss or Gain}

Figure 1 compares the weight of the $M$. oleifera treated rats with a mean of 132.2 \pm 5.05 and the control with a mean value of $134.1 \pm 5.08$ ( $\mathrm{P}>0.8115)$ indicating lack of statistically significant difference in the weight of the rats (tests and controls). This means that $M$. oleifera does not cause increase in fat deposits or sudden weight gain and obesity or weight loss. 


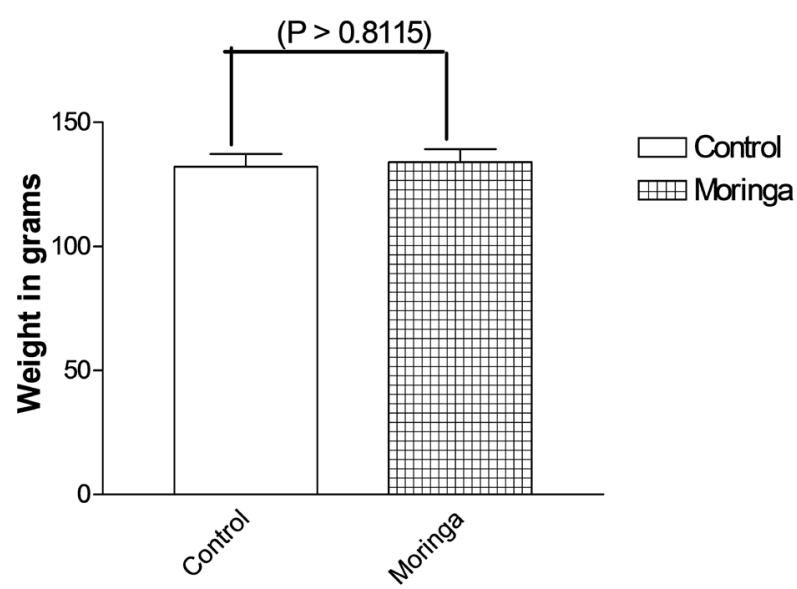

Figure 1. Showing a comparison of the weights of the lipophilic extract of $M$. oleifera fed rats with that of the control; this suggest that they was no statistically significant difference in the weights of the rats.

\subsection{Daily Intake of M. oleifera Extract Did Not Result in Statistically Significant Biochemical Tissue Toxicity, Including Hematological/Pulmonary Endothelial Cell Cytotoxicity}

LDH is most often measured to check for tissue damage. The protein LDH is in many body tissues, especially the heart, liver, kidney, muscles, brain, blood cells, and lungs. LDH significantly correlated with plasma levels of soluble VCAM-1, a marker of endothelial cell activation [13]. In particular elevated levels in LDH are strong indicator of pulmonary toxicity and or toxicity to the red blood cells causing haemolysis [14] [15]. LDH has long been considered a useful clinical marker of intravascular haemolysis [15]. Its serum levels are mildly elevated in extravascular haemolysis, such as immune hemolytic anemia, but are substantially elevated with intravascular haemolysis, such as thrombotic thrombocytopenic purpura and paroxysmal nocturnal hemoglobinuria [14] [15]. To determine the extent of cytotoxicity in extract treated or vehicle treated rats, total LDH concentrations were measured using colorimetric assay kit (Promega, Madison, WI, USA) according to the manufacturer's protocol. Figure 2 is the serum LDH levels of the tests which have a mean value of $170.7 \pm 13.02$; and the controls with a mean of $133.8 \pm 7.17$ ( $\mathrm{P}>0.0698$ shows that there was no statistically significant level of cytotoxicity between Moringa treated and vehicle treated control group.

\subsection{Treatment with M. oleifera Extract Confers Increased Biochemical Hepatocellular Function}

Aminotransferase levels are sensitive indicators of liver-cell injury. In liver with very high cell viability, alanine aminotransferases are normally present at very low levels, the highest level of alanine aminotransferase is in the liver, and levels of this enzyme are accordingly specific indicators of liver injury. Recently, ALT 
has been shown to be the most discriminator among the panels of test for determining the health or viability of hepatocytes in vivo [12] [16], for this reason ALT was measured in both the group that received moringa and the control and the data compared as indicated in material and method. Figure 3 is the serum ALT levels of the animals that received daily dose on moringa, with a mean value of $12.4 \pm 1.2$; and the controls with a mean of $25.6 \pm 5.644(\mathrm{P}<0.01)$ indicating a strong statistically significant difference between the ALT levels of the Moringa treated group and control.

\subsection{Treatment with $M$. oleifera Extract Effectively Enhanced Recovery from Acetaminophen Induced Hepatic Hepatitis}

Acetaminophen-induced hepatotoxicity in rodents is a widely used animal model to assess hepatoprotective activity of new compounds [17]. To confirm

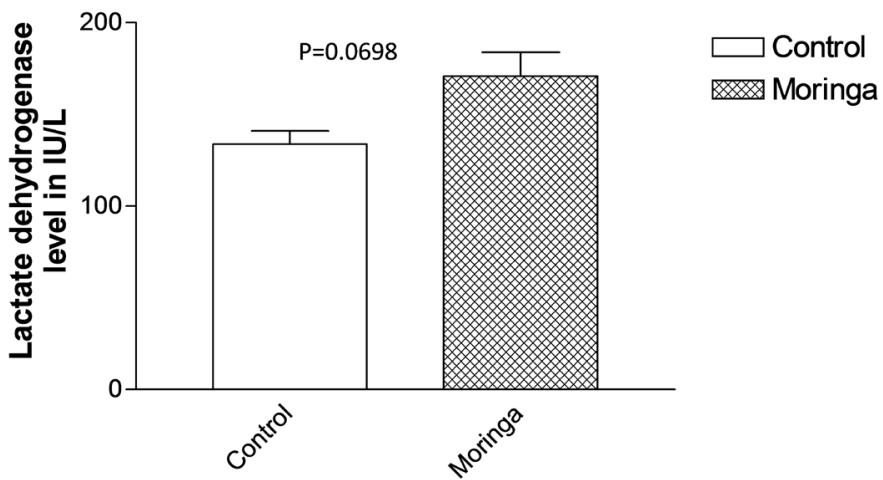

Figure 2. Shows a comparison between the $\mathrm{LDH}$ values of $M$. oleifera fed healthy rats for 14 days to that of the vehicle fed control. Data indicate lack of haematologic/endothelial tissue toxicity; major sources of LDH. Thus there was no statistically significant difference in LDH. However, a long time use is likely to cause and increase in $\mathrm{LDH}$.

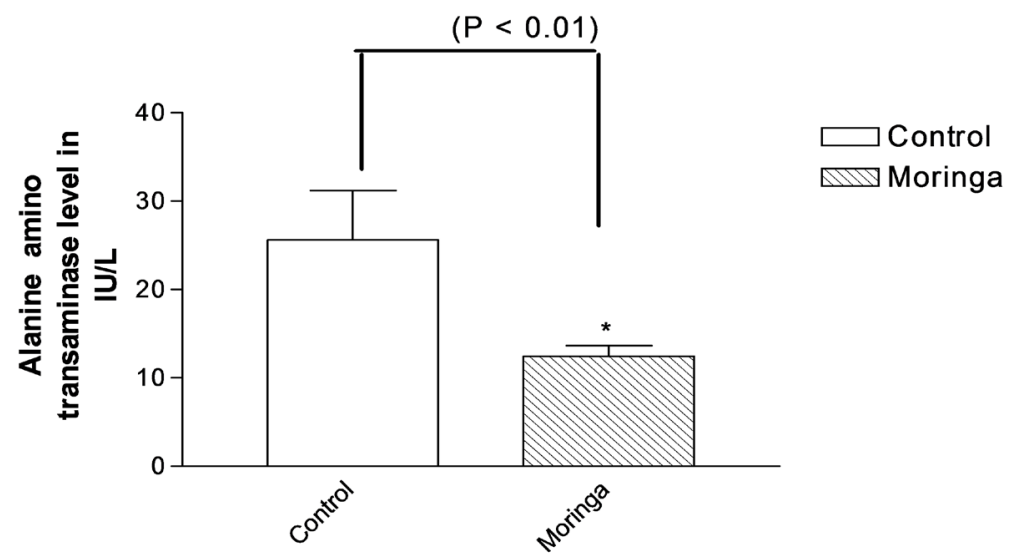

Figure 3. Shows a comparison between the ALT levels of the $M$. oleifera fed healthy rats for 14 days to that of the control; there was a statistically significant decrease in the ALT levels in the rats fed with the lipophilic extract. This means that therapy with lipophilic extract of $M$. oleifera may promote hepatic function and its biochemical robustness. 
the effect seen whenever rats were maintained on a daily dose of Moringa extract, as indicated above, there was a significant increase in hepatocellular viability in health animals. This suggests that Moringa extract may contain active compounds capable allowing liver cell to effectively recover from serious cellular injury. To test this, animals confirmed with drug induced hepatitis were treated with the extract and their liver marker of hepatitis was assessed and compared with the controls that did not receive any treatment except vehicle. Figure 4 compares the level of Alanine aminotransferase (ALT) between the hepatitis group and non hepatitis group. This data confirmed hepatotoxicity or liver cell damage in the group with hepatitis $(\mathrm{P}<0.01)$. There was statistically significant difference between hepatitis group and non-hepatitis group. Figure 5 shows level of alanine aminotransferase (ALT) between groups with hepatitis treated with Moringa and the group with hepatitis treated with vehicle (saline). Data showed that, group treated with moringa, had a statistically significant decrease in the level of alanine aminotransferase after treatment $(\mathrm{P}<0.0006)$. While the group with hepatitis treated with vehicle (saline) had a very high level of alanine aminotransferase.

\section{Discussion}

To date efforts to identify treatment regimen that slow, halt or reverse progressive liver damage or hepatitis have not been successful. We have shown that

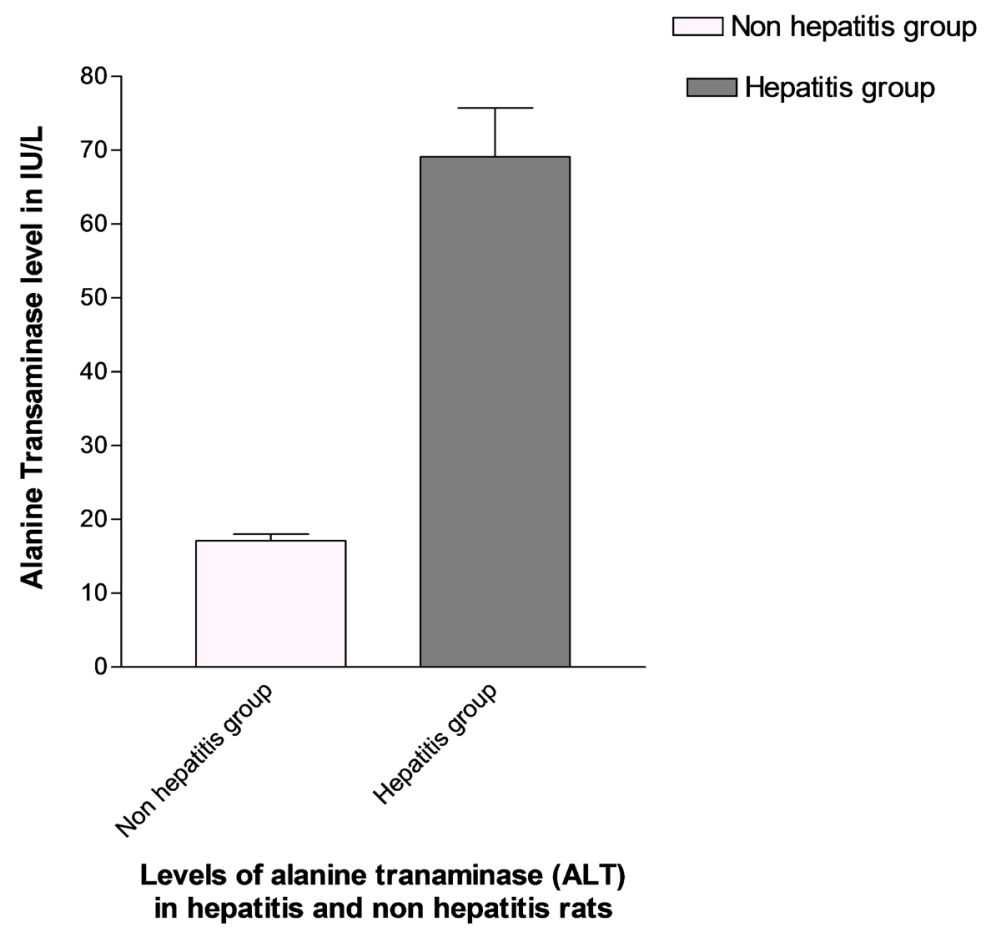

Figure 4. Illustrating the serum levels of ALT of rats that had experimentally induced hepatitis with xenobiotics acetaminophen and the control group that were treat with vehicle only. The data confirmed that rats that had high dose of acetaminophen all developed drug induced hepatitis. 


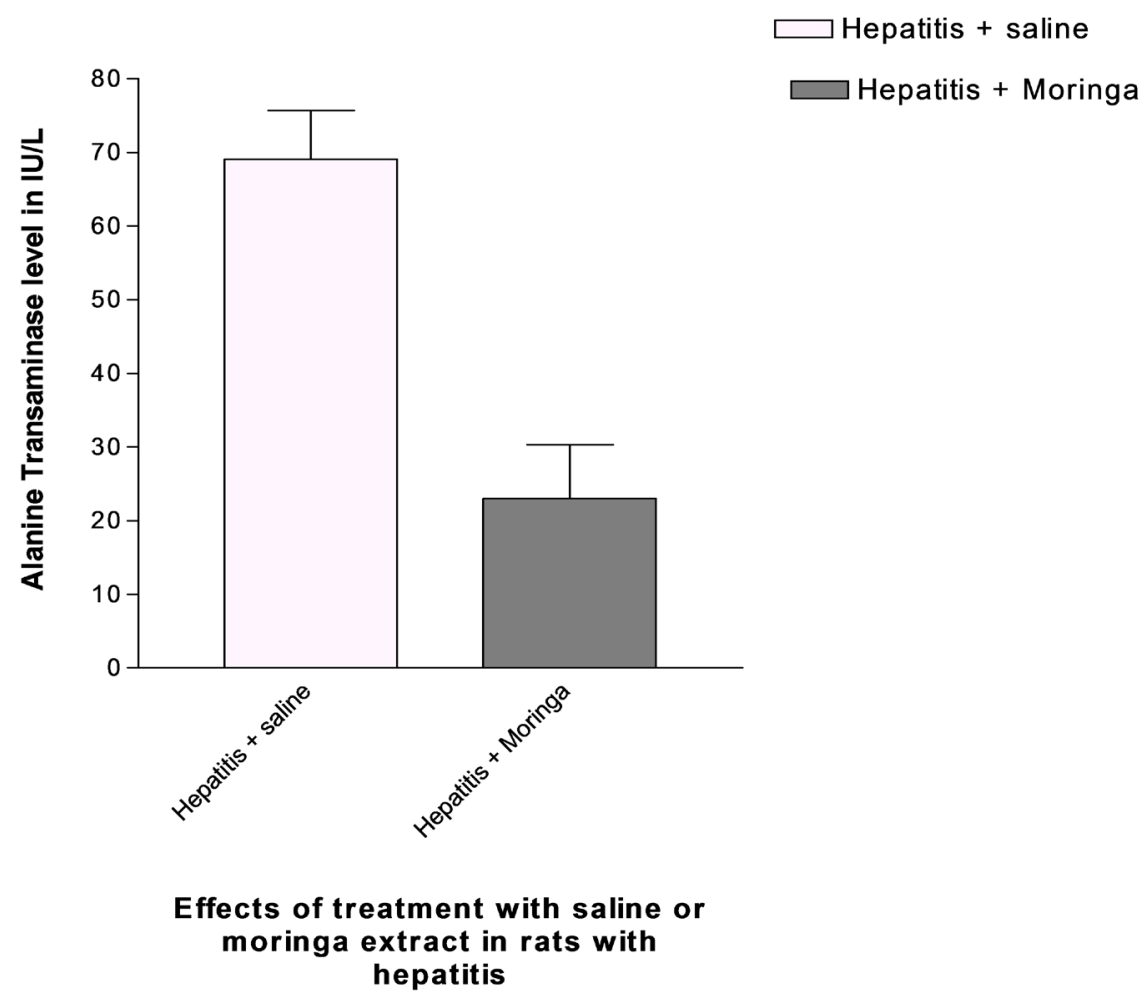

Figure 5. Illustrating the effect of treatment with lipophilic extract of $M$. oleifera on drug induced hepatitis. Rats with confirmed hepatitis received either vehicle or lipophilic extract of $M$. oleifera for 14 days. Treatment with lipophilic extract of $M$. oleifera resulted in decreased serum levels of ALT, and a concomitant return of the serum levels of ALT a baseline levels as found in healthy non hepatitis group. Data represents the mean and standard error of mean of at least 3 independent experiments.

treatment with lipophilic extract of $M$. oleifera in healthy rat did not cause any weight changes in comparison with the control animals that received only the vehicle, Figure 1. Interestingly, observation from acute toxicity experiment suggests lack of changes in satiety, or feeding habit, or changes involving locomotive or physical alertness in the animals shortly after and 14 days of receiving oral dose of the extract, indicating that the extract may not induce neurotoxicity within 14 days of therapy. Data from cytotoxicity experiment further supported this observation. LDH has long been considered a useful clinical marker of intravascular haemolysis. (Hematologic toxicity and, /or pulmonary endothelial toxicity [13] Importantly, whenever healthy animals were exposed to daily therapy with the extract, there was a slight but no statistically significant elevation in LDH level, suggesting lack of haematological/endothelial toxicity for preclinical use of the extract (Figure 2). This data is in line with a previous report of Tako et al. [13]. However, there was a slight elevation of LDH in the extract treated healthy rats, which was not statistically significant; this may indicate a long time effect for use of the extract, however, the physiological interpretation needs to be confirmed.

Of note was that therapy with the extract resulted in reduced ALT level com- 
pared with the controls in healthy rats (Figure 3), suggesting that the extract may contain crude substances that may confer liver with increased functional robustness. This is in line with the report of Oyagbemi et al., [18] who observed that treatment with extract of $M$. oleifera was associated with reduced hepatic injury. Figure 6; a schematic representation suggesting that treatment with hydrophilic extract of Moringa oleifera Lam, may abrogate xenobiotics induced liver damage and hepatitis in rat.

Hepatitis is a condition defined by the inflammation of the liver and it's seen in drug induced or viral hepatitis. Regardless of the underlying cause, hepatotoxicity or liver cell injury causes inflammatory damage, matrix deposition, parenchymal cell death (Figure 6) and angiogenesis leading to progressive liver failure [19]. Once cirrhosis is established the potential for reversing this process is decreased and complications develop [3] [19], however, certain small molecules can stimulate progenitor cells leading to proliferation and differentiation which can result in accurate tissue repair [20]. We have shown that drug induced hepatotoxicity in rat (Figure 4$)$ was reverted or repaired $(\mathrm{P}<0.0006)$ by treatment with $M$. oleifera extract for 14 days compared with control (Figure 4 and Figure 5). The current data strongly suggests that lipophilic extracts of $M$. oleifera may promote liver resilience and enhance recovery from xenobiotics-induced hepatitis or hepatotoxicity (Figure 6). It is likely some components of the extract may have stimulatory effects that induced proliferation of the resident progenitor cells in the liver, which proliferated and repaired the effect of xenobiotics induced liver damage during the course of treatment with the extract. In addition, it is known that liver injury causes parenchymal cell necrosis and/or apoptosis; the release of cell contents and reactive oxygen species (ROS) activates hepatic stellate cells and attracts and activates tissue macrophages

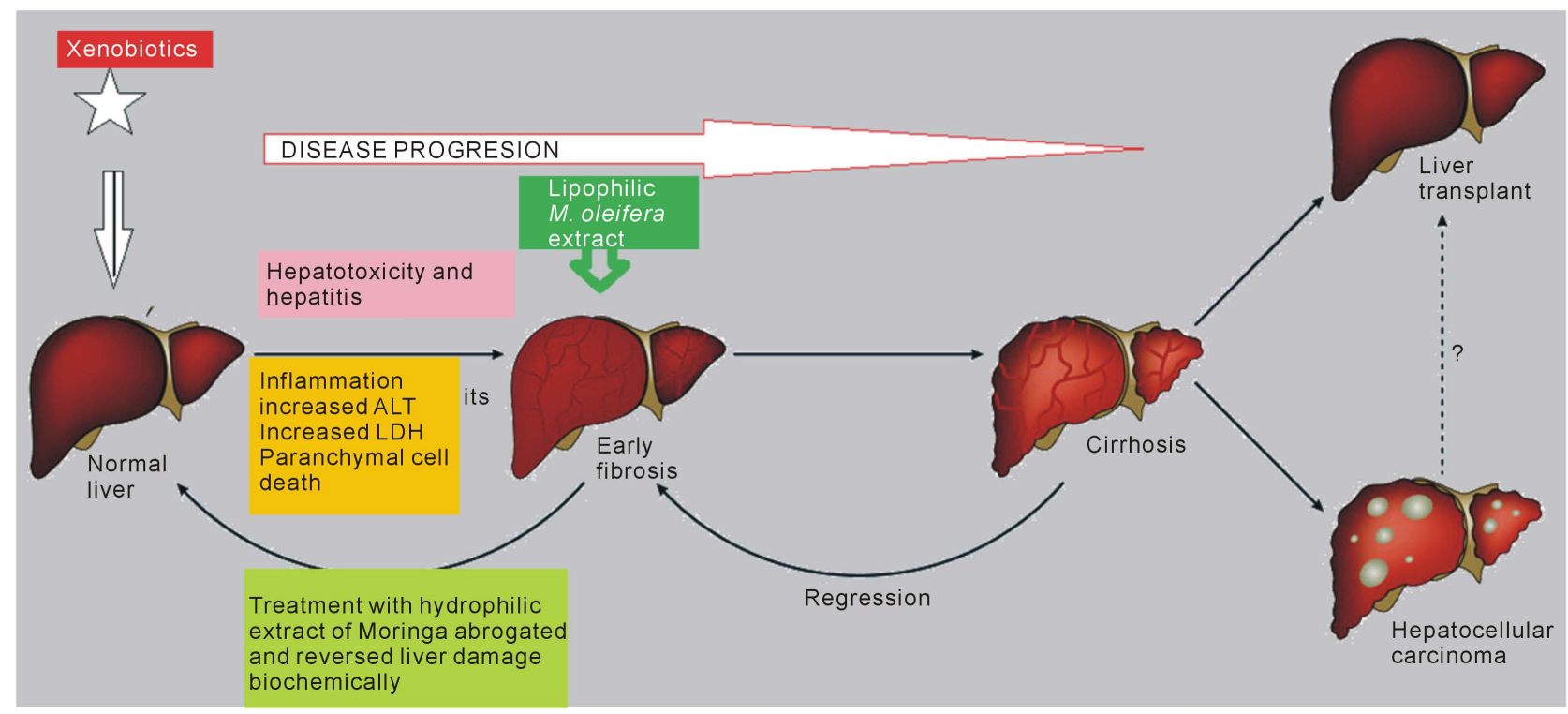

Figure 6. A schematic representation suggesting that treatment with hydrophilic extract of Moringa oleifera Lam, may abrogate xenobiotics induced liver damage and hepatitis in rat. 
through the CC-chemokines ligand 2 (CCL2)-CC-chemokine receptor 2 (CCR2) [3] [19]. Recent study indicated that $M$. oleifera preparations have many active antioxidant, including vitamins $\mathrm{C}, \mathrm{E}$, and $\mathrm{A}$; and essential micronutrients such as selenium and zinc and [21]. Perhaps these may explain the physiology of the current findings of this study.

In line with the finding of this study, Sheikh et al., [22] showed that food supplementation with $M$. oleifera leaves abrogated arsenic-induced elevation of triglyceride, glucose, urea and the activities of alkaline phosphatase, aspartate aminotransferase and alanine aminotransferase in serum. Like in the current studies, orally administered $M$. oleifera leaves blocked various toxic effects of arsenic in mice [22]. For the first time, we have demonstrated that drug induced hepatotoxicity or hepatitis was abrogated and reversed by treatment with extract of $M$. oleifera. These pre-clinical data are promising for therapeutic application of $M$. oleifera extract in treating hepatitis including drug induced liver damage in rat, and points to further research for a possible application in clinical setting and in patients with abnormally elevated liver enzymes [23] [24]. At this point, the mechanism by which treatment with the lipophilic extract of $M$. oleifera reversed liver damage is not known; therefore, further studies may investigate the mechanism and also isolate the active substances contained in the crude extract.

\section{Conclusion}

In conclusion, we have shown that xenobiotics induced hepatotoxicity was completely reversed to a normal level of liver function enzymes following treatment with extract from $M$. oleifera. This study strongly indicates that therapy and treatment with lipophilic extract of $M$. oleifera effectively and biochemically abrogated xenobiotics induced liver damage in animal model, indicating that the hydrophilic $M$. oleifera extract may contain bioactive compounds with potential to slow; halt or reverse progression of drug induced hepatitis in rat. These findings may help us better understand the mechanisms of xenobiotics induced hepatotoxicity regarding the reported constituents of this extract as well as in developing novel therapy strategies for treating liver disease especially in resource limited countries.

\section{Conflict of Interest}

The authors declare there are no conflict of interest, and that no fund from any funding body was made available for this work. This study was funded by the authors only.

\section{References}

[1] Dechêne, A., Sowa, J.P., Gieseler, R.K., et al. (2010) Acute Liver Failure Is Associated with Elevated Liver Stiffness and Hepatic Stellate Cell Activation. Hepatology, 52, 1008-1016. https://doi.org/10.1002/hep.23754

[2] O’Grady, J.G. (2005) Acute Liver Failure. Postgraduate Medical Journal, 81, 148-154. https://doi.org/10.1136/pgmj.2004.026005 
[3] Pellicoro, A., Ramachandran, P., Iredale, J.P., et al. (2014) Liver Fibrosis and Repair: Immune Regulation of Wound Healing in a Solid Organ. Nature Reviews Immunology, 3, 181-194. https://doi.org/10.1038/nri3623

[4] Bourbonnais, E., Raymond, V.A., Ethier, C., et al. (2012) Liver Fibrosis Protects Mice from Acute Hepatocellular Injury. Gastroenterology, 142, 130-139. https://doi.org/10.1053/j.gastro.2011.09.033

[5] Protzer, U., Maini, M.K. and Knolle, P.A. (2012) Living in the Liver: Hepatic Infections. Nature Reviews Immunology, 12, 201-213. https://doi.org/10.1038/nri3169

[6] Omabe, M., Nwudele, C., Omabe, K.N., et al. (2014) Anion Gap Toxicity in Alloxan Induced Type 2 Diabetic Rats Treated with Antidiabetic Noncytotoxic Bioactive Compounds of Ethanolic Extract of Moringa oleifera. Journal of Toxicology, 2014, Article ID: 406242. https://doi.org/10.1155/2014/406242

[7] Omabe, M. and Kenneth, N.O. (2014) Insight in Inflammation and Cancer. The Atlas of Genetics and Cytogenetics in Oncology and Haematology, 18, 203-216. https://doi.org/10.4267/2042/53491

[8] Ghasi, S., Nwobodo, E. and Ofili, J.O. (2000) Hypocholesterolemic Effects of Crude Extract of Leaf of $M$. oleifera Lam in High-Fat Diet Fed Wistar Rats. Journal of Ethnopharmacology, 69, 21-25. https://doi.org/10.1016/S0378-8741(99)00106-3

[9] Lee, S.W., Kim, S.H., Min, S.O. and Kim, K.S. (2011) Ideal Experimental Rat Models for Liver Diseases. Korean Journal of Hepato-Biliary-Pancreatic Surgery, 15, 67-77. https://doi.org/10.14701/kjhbps.2011.15.2.67

[10] Workman, P., Aboagye, E.O., Balkwill, F., et al. (2010) Guidelines for the Welfare and Use of Animals in Cancer Research. British Journal of Cancer, 102, 1555-1577. https://doi.org/10.1038/sj.bjc.6605642

[11] Baravalia, Y. and Chanda, S. (2011) Protective Effect of Woodfordia fruticosa Flowers against Acetaminophen-Induced Hepatic Toxicity in Rats. Pharmaceutical Biology, 49, 826-832. https://doi.org/10.3109/13880209.2010.550057

[12] Navarro, V.J. and Senior, J.R. (2006) Drug-Related Hepatotoxicity. The New England Journal of Medicine, 354, 731-739. https://doi.org/10.1056/NEJMra052270

[13] Kato, G.J., McGowan, V., Machado, R.F., et al. (2006) Lactate Dehydrogenase as a Biomarker of Hemolysis-Associated Nitric Oxide Resistance, Priapism, Leg Ulceration, Pulmonary Hypertension, and Death in Patients with Sickle Cell Disease. Blood, 107, 2279-2285. https://doi.org/10.1182/blood-2005-06-2373

[14] Kato, G.J., Martyr, S., Blackwelder, W.C., et al. (2005) Levels of Soluble Endothelium-Derived Adhesion Molecules in Patients with Sickle Cell Disease Are Associated with Pulmonary Hypertension, Organ Dysfunction, and Mortality. British Journal of Haematology, 130, 943-953.

https://doi.org/10.1111/j.1365-2141.2005.05701.x

[15] Tabbara, I.A. (1992) Hemolytic Anemias. Diagnosis and Management. Medical Clinics of North America, 76, 649-668. https://doi.org/10.1016/S0025-7125(16)30345-5

[16] Lilford, R.J., Bentham, L., Girling, A., et al. (2013) Birmingham and Lambeth Liver Evaluation Testing Strategies (BALLETS): A Prospective Cohort Study. Health Technology Assessment, 17, 1-307. https://doi.org/10.3310/hta17280

[17] Ramachandran, R. and Kakar, S. (2009) Histological Patterns in Drug-Induced Liver Disease. Journal of Clinical Pathology, 62, 481-492. https://doi.org/10.1136/jcp.2008.058248

[18] Oyagbemi, A.A., Omobowale, T.O., Azeez, I.O., et al. (2013) Toxicological Evalua- 
tions of Methanolic Extract of $M$. oleifera Leaves in Liver and Kidney of Male Wistar Rats. Journal of Basic and Clinical Physiology and Pharmacology, 4, 307-312. https://doi.org/10.1515/jbcpp-2012-0061

[19] Bordon, Y. (2014) Microbiota: The Liver Debugs the System. Nature Reviews Immunology, 14, 430-431. https://doi.org/10.1038/nri3704

[20] Boulter, L., Lu, W.Y. and Forbes, S.J. (2013) Differentiation of Progenitors in the Liver: A Matter of Local Choice. The Journal of Clinical Investigation, 123, 1867-1873. https://doi.org/10.1172/JCI66026

[21] Abd-El Latif, A., El Bialy Bel, S., Mahboub, H.D., et al. (2014) M. oleifera Leaf Extract Ameliorates Alloxan-Induced Diabetes in Rats by Regeneration of $\beta$ Cells and Reduction of Pyruvate Carboxylase Expression. Biochemistry and Cell Biology, 92, 413-419. https://doi.org/10.1139/bcb-2014-0081

[22] Sheikh, A., Yeasmin, F., Agarwal, S., et al. (2014) Protective Effects of M. oleifera Lam. Leaves against Arsenic-Induced Toxicity in Mice. Asian Pacific Journal of Tropical Biomedicine, 1, 353-358. https://doi.org/10.12980/APJTB.4.201414B44

[23] Pratt, D.S. and Kaplan, M.M. (2000) Evaluation of Abnormal Liver-Enzyme Results in Asymptomatic Patients. The New England Journal of Medicine, 342, 1266-1271. https://doi.org/10.1056/NEJM200004273421707

[24] Iwaisako, K., Jiang, C., Zhang, M., et al. (2014) Origin of Myofibroblasts in the Fibrotic Liver in Mice. Proceedings of the National Academy of Sciences, 111, 3297-3305. https://doi.org/10.1073/pnas.1400062111 